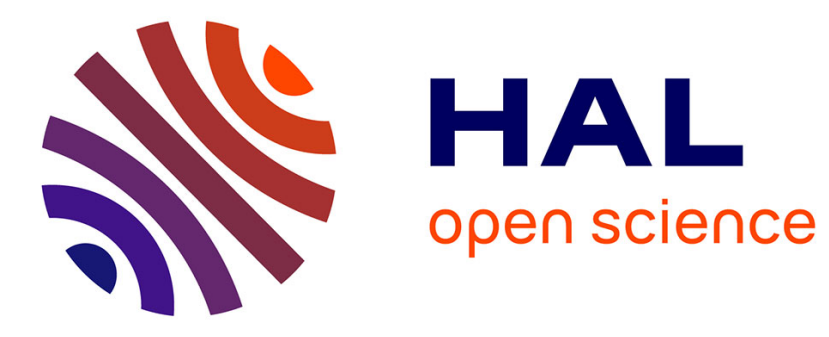

\title{
Low electrical percolation thresholds and nonlinear effects in graphene-reinforced nanocomposites: a numerical analysis
}

Xiaoxin Lu, Julien Yvonnet, Fabrice Detrez, Jinbo Bai

\section{- To cite this version:}

Xiaoxin Lu, Julien Yvonnet, Fabrice Detrez, Jinbo Bai. Low electrical percolation thresholds and nonlinear effects in graphene-reinforced nanocomposites: a numerical analysis. Journal of Composite Materials, inPress, accepted, 10.1177/0021998317753888 . hal-01674146

\section{HAL Id: hal-01674146 \\ https://hal.science/hal-01674146}

Submitted on 1 Jan 2018

HAL is a multi-disciplinary open access archive for the deposit and dissemination of scientific research documents, whether they are published or not. The documents may come from teaching and research institutions in France or abroad, or from public or private research centers.
L'archive ouverte pluridisciplinaire HAL, est destinée au dépôt et à la diffusion de documents scientifiques de niveau recherche, publiés ou non, émanant des établissements d'enseignement et de recherche français ou étrangers, des laboratoires publics ou privés. 


\title{
Low electrical percolation thresholds and nonlinear effects in graphene-reinforced nanocomposites: a numerical analysis
}

\author{
Xiaoxin $\mathrm{Lu}^{\mathrm{a}, \mathrm{b}}$, Julien Yvonnet ${ }^{\mathrm{b}, *}$, Fabrice Detrez ${ }^{\mathrm{b}}$, Jinbo Bai ${ }^{\mathrm{a}, * *}$ \\ ${ }^{a}$ Centrale Supélec, Laboratoire de Mécanique des Sols, Structures et Matériaux, \\ UMR 8579 CNRS, \\ Grande Voie des Vignes, 92290 Châtenay Malabry, France \\ ${ }^{b}$ Université Paris-Est, Laboratoire de Modélisation et Simulation Multi Echelle, \\ UMR 8208 CNRS, \\ 5 Boulevard Descartes, 77454 Marne-la-Vallée Cedex 2, France
}

\begin{abstract}
A numerical model of graphene-reinforced nanocomposites taking into account the electric tunneling effect is employed to analyze the influence of microstructural parameters on the effective electric conductivity and the percolation thresholds of the composite. The generation procedure for the random microstructures of graphene reinforced nanocomposites is described. Effects of the barrier height, of graphene aspect ratio and alignment of graphene sheets have been quantitatively evaluated. The results show that both higher graphene aspect ratio and lower barrier height can lead to smaller percolation threshold, and the alignment of graphene sheets results in anisotropic electrical behavior without affecting the percolation threshold. The numerical model also shows the importance of the tunneling effect to reproduce the nonlinear electric behavior and the low percolation thresholds reported in the literature. Finally, results are compared with available experimental data.
\end{abstract}

Keywords:

Nanocomposites, Graphene, Electric conduction, Tunnel effect, Percolation threshold

*Corresponding author Email: julien.yvonnet@univ-paris-est.fr

${ }^{* *}$ Corresponding author Email: jinbo.bai@ecp.fr 


\section{Introduction}

The introduction of graphene-based nanomaterials has prompted the development of flexible nanocomposites for emerging applications in various fields, including energy conversion [1], energy storage [2], electronic materials [3], sensors [4], and chemical screening applications [5]. Graphene is among the materials with the highest in-plane electric conductivity [6], and its incorporation in a polymer matrix to increase the electric conductivity of almost insulating polymers is of high importance for materials-by-design. Numerous experimental and theoretical studies have reported that dispersing two-dimensional fillers such as graphene sheets in polymer matrix can significantly improve the electric properties of the resulting composites [7-12]. They exhibit an increase of several orders of magnitude of the electric conductivity even at extremely low volume fractions of graphene sheets [13-17], denoting the percolation phenomenon. This phenomenon can be explained by the formation of percolating paths between graphene sheets through the matrix. The percolation threshold of a random assembly of widthless discs has been evaluated in last century for the permeability problem [18], dominated by the radius and the number of the discs. However, the introduction of nano fillers brings more complexity to the study of connectivity percolation of the composite. Mathew et al. [19] have presented Monte Carlo simulation study on the percolation in a random array of 3D impenetrable hard platelets, revealing the influence of the filler's aspect ratio.

Experimental works have evidenced that electrical properties of graphene or nanotube-reinforced nanocomposites are significantly dependent on the microstructure parameters such as the size [20], the orientation of fillers [17, 21], as well as the inherent characteristics of polymer matrix [22, 23].

Tunneling electric conductivity is a quantum phenomenon which allows electric conductivity across small isolating barriers like thin polymer interphases between two highly conducting fillers. Especially when the characteristic distances between graphene sheets reduces to the order of nanometers, it has been shown that electric tunneling effect plays an important role in explaining very low percolation thresholds as well as nonlinear electric conduction $[24,25]$ in such materials. In the works of Otten et al. [26] and Ambrosetti et al. [27], the tunneling effect has been taken into account to analyze the percolation threshold of nanocomposites with polydisperse nanofillers, where the former focuses on carbon nanotube and the latter extends to various particle shapes. 
For design purpose, numerical models of conduction at the scale of a Representative Volume Element (RVE) containing a significant number of graphene sheets is required to fully understand the conditions for percolation and determining optimal configurations and microstructural parameters to increase the performances of these materials. Complete ab initio or atomistic simulations including electric conductivity in large systems like polymers-graphene reinforced composites are nowadays not feasible, and classical homogenization methods or Monte Carlo techniques [28-34] are unable to explain the nonlinear effects and low percolation thresholds in graphenepolymer nanocomposites. Simulations of larger systems require continuum description of fields and related numerical methods. Recently, some continuum models have been developed to determine the effective AC and DC electrical properties of graphene nanocomposites taking into account their morphological and physical features, which take the effective-medium theory or self-consistent effective medium theory as backbone [31, 35, 36]. A multi-scale multi-physics finite element method has also been proposed to predict the electrical response of the graphene/polymer composite under DC loading, in which the representative volume element (RVE) is filled with randomly dispersed nano-platelet conductive inclusions by Monte Carlo model and with the use of a unit cell [37].

In this work, we employ the continuum numerical tunneling model framework introduced in [38] to quantitatively analyze the effects of barrier height and configuration of graphene sheets versus electric percolation thresholds in graphene-polymer nanocomposites. This methodology provides the possibility to analyze the influence of these various factors on the electrical conductivity as well as the percolation threshold separately, and can be easily extended to investigate the effect of different graphene distributions. First, the continuum model is presented taking into account the tunneling effect, in which the graphene is modeled as a highly conductive surface in order to avoid the meshing restrictions. Then, the geometrical modeling of graphene/polymer nanocomposites is provided. The generation of the random microstructure is implemented by Markov chains method [39, 40]. According to the numerical analysis, the nonlinear electric behavior of the graphene reinforced nanocomposite is characterized, and the effective conductivities of nanocomposites with various barrier height between graphene and polymer as well as different graphene aspect ratio are studied. In addition, the effect of alignment of graphene sheets with respect to the effective conductivity is investigated. Finally, we provide comparisons between the results provided by the pro- 
posed model and some available experimental data. In the present work, the influence of the defects (e.g. nanopres) in the composite is neglected, and the aggregation of graphene fillers is not taken into account.

\section{Continuum model}

In this model, the graphene sheets are explicitly described at the scale of a Representative Volume Element (RVE) composed of a polymer matrix and randomly distributed and oriented graphene sheets, modeled as highly conducting imperfect interfaces [41]. Another originality of the mentioned work is the modeling of the electric tunneling effect between graphene sheets.

In this model, the total electric power of the system, $\bar{W}$, is defined by

$$
2 \bar{W}=\int_{\Omega} \omega^{b}(\mathbf{x}) d \Omega+\int_{\Gamma} \omega^{s}(\mathbf{x}) d \Gamma,
$$

where $\Omega \subset \mathbb{R}^{3}$ is the domain defining the RVE, $\Gamma$ denotes collectively the surfaces associated with graphene and the density functions $\omega^{b}$ and $\omega^{s}$ are expressed by

$$
\omega^{b}(\mathbf{x})=j_{i}(\mathbf{x}) E_{i}(\mathbf{x}), \quad \omega^{s}(\mathbf{x})=j_{i}^{s}(\mathbf{x}) E_{i}^{s}(\mathbf{x}),
$$

where $E_{i}(\mathbf{x})=-\nabla_{i} \phi(\mathbf{x})=-\phi_{, i}(\mathbf{x})$ is the electric field, $\mathbf{j}(\mathbf{x})$ is the current density vector and $\phi(\mathbf{x})$ is the electric potential, where:

$$
j_{i}=\left[k_{p}^{0}\right]_{i j} E_{j}
$$

with $\mathbf{k}_{p}^{0}$ is the electric conductivity tensor of the polymer matrix when neglecting tunneling effect. In the above, the superscript $s$ denotes surface quantities, e.g., $\mathbf{j}^{s}$ is the surface current density. The surface electric field is defined with respect to its bulk counterpart as: $E_{i}^{s}=P_{i j} E_{j}=-\nabla_{i}^{s} \phi=$ $-P_{i j} \nabla_{i} \phi$ with $P_{i j}(\mathbf{x})=\delta_{i j}-n_{i}(\mathbf{x}) n_{j}(\mathbf{x})$ is a projector operator characterizing the projection of a vector along the tangent plane to $\Gamma$ at a point $\mathbf{x} \in \Gamma$ and $\mathbf{n}$ is the unit normal vector to $\Gamma$. The local constitutive relationships relating $\mathbf{j}$ and $\mathbf{j}^{s}$ with $\mathbf{E}$ are defined in the graphene sheets by

$$
j_{i}^{s}(\mathbf{x})=k_{i j}^{s} E_{j}^{s}
$$


where $\mathbf{k}^{s}$ denotes the surfacic electric conductivity of graphene and is dependent on the thickness $t$ through:

$$
k_{i j}^{s}=t S_{i j}, \quad S_{i j}=\left[k^{g}\right]_{i j}-\frac{\left[k^{g}\right]_{i k} n_{k}\left[k^{g}\right]_{j k} n_{k}}{\left[k^{g}\right]_{k l} n_{k} n_{l}} .
$$

In (5), $\mathbf{k}^{g}$ denotes the second-order electric conductivity tensor of the bulk graphite and $\mathbf{n}$ the normal vector to the graphene sheet (see $[38,41]$ for more details).

$$
j_{i}= \begin{cases}{\left[k_{p}^{0}\right]_{i j} E_{j}} & \text { if } d(\mathbf{x}) \geq d_{c u t}, \\ \mathcal{G}(\mathbf{E}, d) \frac{E_{i}}{|\mathbf{E}|} & \text { if } d(\mathbf{x})<d_{c u t},\end{cases}
$$

where $d_{c u t}$ is a cut-off distance above which the tunneling effect can be neglected and $\mathcal{G}$ is defined by [42]

$$
\begin{aligned}
& \mathcal{G}(\mathbf{E}, d)=\frac{2.2 e^{3}|\mathbf{E}|^{2}}{8 \pi h \Phi_{0}} \exp \left(-\frac{8 \pi}{2.96 h e|\mathbf{E}|}(2 m)^{\frac{1}{2}} \Phi_{0}^{\frac{3}{2}}\right) \\
& +\left[3 \cdot \frac{\left(2 m \Phi_{0}\right)^{\frac{1}{2}}}{2}\right](e / h)^{2}|\mathbf{E}| \exp \left[-\left(\frac{4 \pi d}{h}\right)\left(2 m \Phi_{0}\right)^{\frac{1}{2}}\right]
\end{aligned}
$$

where $\Phi_{0}$ is the energy barrier height that the electrons cross and $h, e$ and $m$ denote Plank's constant, the charge of an electron and a material parameter.

Minimizing (1) with respect to the displacement field, and using Eqs. (3)-(6), we obtain the weak form which can be solved by the finite element method (see more details in [38]):

$$
\int_{\Omega} j_{i}(\phi) \cdot \nabla(\delta \phi) d \Omega-\int_{\Gamma} P_{i j} \nabla_{j} \phi k_{i k}^{s} P_{k l} \nabla_{l}(\delta \phi) d \Gamma=0
$$

where $\delta \phi \in H^{1}(\Omega), \delta \phi=0$ over $\partial \Omega$, and $\phi \in H^{1}(\Omega)$, $\phi$ satisfying the periodic boundary conditions over $\partial \Omega$

$$
\phi(\mathbf{x})=-\bar{E}_{i} x_{i}+\tilde{\phi}(\mathbf{x}) \quad \text { on } \partial \Omega
$$

and where $\tilde{\phi}(\mathbf{x})$ is a periodic function over $\Omega$, such as $\langle\tilde{\phi}(\mathbf{x})\rangle=0$.

The effective electric conductivity tensor $\overline{\mathbf{k}}$ is defined as:

$$
\bar{k}_{i j}(\overline{\mathbf{E}})=\frac{\partial \bar{J}_{i}(\overline{\mathbf{E}})}{\partial \bar{E}_{j}}
$$




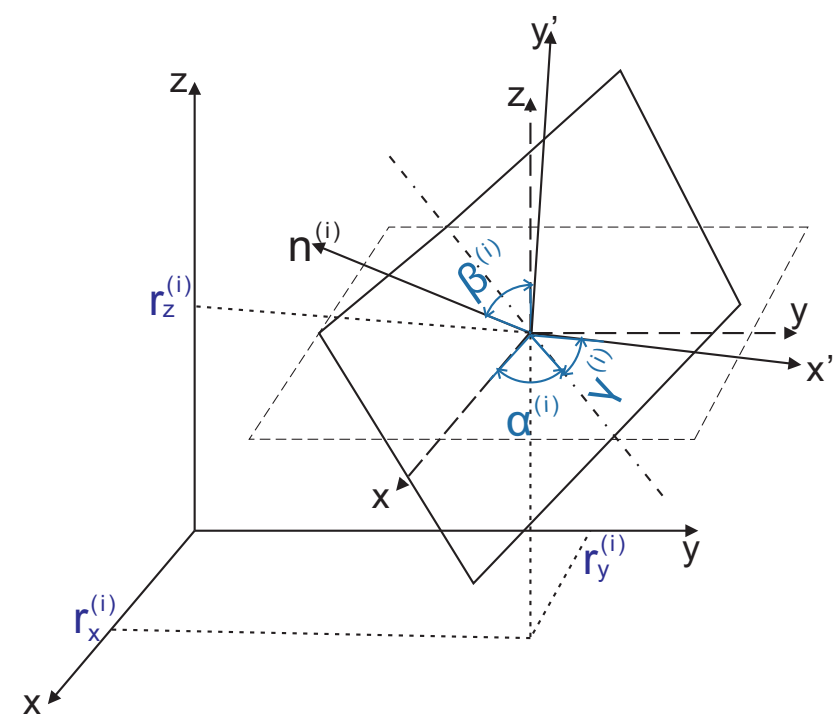

Figure 1: Scheme of the position and oriention of graphene sheet in the 3D space.

where $\overline{\mathbf{J}}$ is the effective current density whose components are expressed by:

$$
\bar{J}_{i}=\frac{1}{V}\left(\int_{\Omega} j_{i}(\mathbf{x}) d \Omega+\int_{\Gamma} j_{i}^{s}(\mathbf{x}) d \Gamma\right)
$$

and $\overline{\mathbf{E}}$ is the effective electric field given by:

$$
\bar{E}_{i}=\frac{1}{V} \int_{\Omega} E_{i}(\mathbf{x}) d \Omega .
$$

\section{Geometrical modeling of graphene/polymer nanocomposites}

In the following, the in-plane dimensions of graphene sheets are $15 \times 15$ $n m^{2}$ and the RVE side length of the cubic domain is $80 \mathrm{~nm}$. We consider multi-layer graphene platelets, also called here sheets, which have a finite thickness $t$. The related aspect ratio $\eta=L / t$ denotes the ratio between the length of the platelet and its thickness. It is worth noting that changing the aspect ratio does not change the discretization here, as $t$ only changes in Eq. (5). The position and orientation of $i$ th graphene sheet in RVE are defined by 6 degrees of freedom $\left\{r_{x}^{(i)}, r_{y}^{(i)}, r_{z}^{(i)}, \alpha^{(i)}, \beta^{(i)}, \gamma^{(i)}\right\}$ where $r_{x}^{(i)}, r_{y}^{(i)}, r_{z}^{(i)}$ are the coordinates of the center of graphene sheet, and $\alpha^{(i)}, \beta^{(i)}, \gamma^{(i)}$ are the Euler 
angles. Note that $\alpha^{(i)}, \beta^{(i)}$ give the orientation of the unit normal of graphene sheet, $n^{(i)}$, and $\gamma^{(i)}$ gives the orientation of graphene sheet around the unit normal $n^{(i)}$ (see Figure 1). In this study, the graphene sheets are modeled by square planes with side length $L=15 \mathrm{~nm}$. This assumption is due to the highly thin thickness of graphene and relies on the imperfect surface model.

The different microstructures are generated by a Markov-chain with hardinclusion Metropolis algorithm (see [39, 40] for review in the case hardspheres). First, an initial configuration of the system is chosen such that graphene sheet centers are located on a cubic lattice and have random orientation without overlapping. Then, one randomly chosen graphene sheet is attempted to move for each step of Metropolis algorithm. A new position for the graphene sheet under consideration is chosen by six random parameters: $(\Delta x, \Delta y, \Delta z) \in\left[-\delta_{x}, \delta_{x}\right]^{3}, \Delta \alpha \in[-\pi, \pi], \Delta \beta \in\left[-\delta_{\beta}, \delta_{\beta}\right]$ and $\Delta \gamma \in\left[-\delta_{\gamma}, \delta_{\gamma}\right]$. The parameter $(\Delta x, \Delta y, \Delta z, \Delta \gamma)$ and are generated with an uniform distribution over their definition domains and the couple of increment angles $(\Delta \alpha, \Delta \beta)$ are generate with an uniform distribution on the part of the unit sphere defined by $\delta_{\beta}$. The new position of graphene sheet is then defined by $\left(x^{(i)}+\Delta x, y^{(i)}+\Delta y, z^{(i)}+\Delta z, \alpha^{(i)}+\Delta \alpha, \beta^{(i)}+\Delta \beta, \gamma^{(i)}+\Delta \gamma\right)$. The parameters $\delta_{x}, \delta_{\beta}$ and $\delta_{\gamma}$ are adjusted to give about $50 \%$ acceptance of the new position. Periodic boundary conditions are employed throughout the simulation.

Therefore, the acceptance step of this Metropolis algorithm reduces to checking that this displacement does not cause overlap between graphene sheets. To control the overlapping, the graphene sheets are discretized by a compact centered square lattice of small fictitious spherical particles with radius $\delta$. The non-overlapping condition is

$$
r_{i j}^{2} \geq \delta^{2} \quad \forall i, j
$$

where $r_{i j}$ is a distance between the fictitious spherical particles $i$ and $j$.

To generate a series of independent isotropic RVE samples as random maps, the positions and orientations are saved during a Markov-chain sampling with regular interval to ensure the statistic independence of two RVEs. Note that the first RVE is saved when the orientation of the unit normal of graphene sheets is isotropic in an average sense. One example of obtained realization of isotropic graphene nanocomposite RVE is illustrated in Fig. 2. , where 25 graphene sheets are distributed randomly in an RVE, and where the size of each graphene platelet is $15 \times 15 \times 0.3 \mathrm{~nm}^{3}$, and the side length of the platelets is $80 \mathrm{~nm}$, leading to a volume fraction is $0.33 \mathrm{vol} \%$. Finally 




Figure 2: RVE for the graphene/polymer nanocomposites involving 25 graphene sheets $\left(15 \times 15 \times 0.3 \mathrm{~nm}^{3}\right)$ in a cube of $80 \times 80 \times 80 \mathrm{~nm}^{3}$. The graphene volume fraction is 0.33 vol\%.

to generate RVE with aligned graphene sheets, the unit normal of graphene are fixed to be parallel to the $z$-axis (see Fig. 7 (b)).

\section{Numerical analysis and discussion}

\subsection{Nonlinear electric behavior of graphene reinforced nanocomposite}

We first use our simulation model to investigate the influence of the tunneling effect for explaining the nonlinear electric behavior of graphene reinforced nanocomposites. An RVE containing randomly distributed graphene sheets is considered with $1.05 \mathrm{vol} \%$ and $\eta=50$. The barrier height between graphene and PMMA is $\Phi_{0}=0.17 \mathrm{eV}$ [43]. Fig 3 (a) shows the currentdensity- electric field curve, which exhibits a linear (Ohmic) behavior for low applied electric fields, and a nonlinear behavior for higher electric fields. The numerical simulations are in qualitative agreement with the experimental results reported in [44]. 


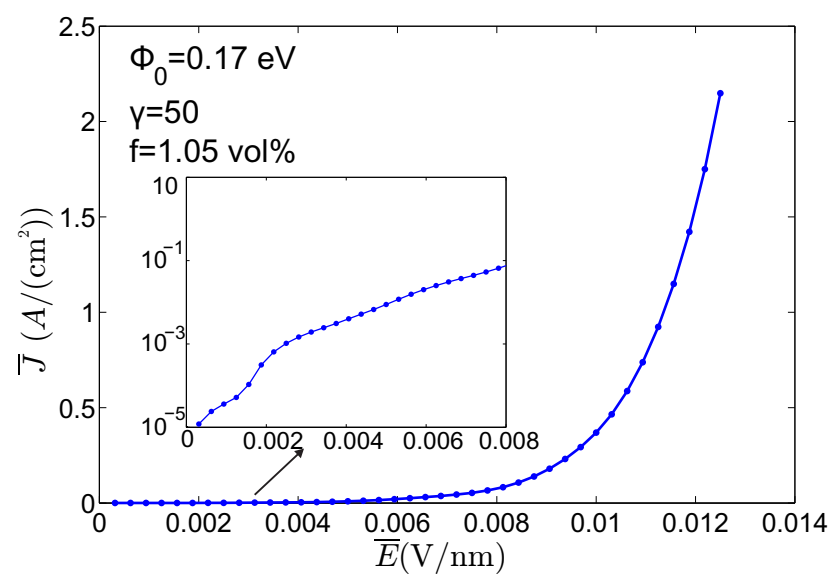

Figure 3: Current density-electric field curve of the nanocomposite with 1.05 vol\% graphene, $\Phi_{0}=0.17 \mathrm{eV}, \eta=50$.

\subsection{Effect of barrier height on the percolation threshold}

In the following, we study the effects of barrier height between graphene and various polymers on the nonlinear response of the nanocomposite. In our simulations, we have considered that the percolation threshold corresponds to a sharp variation of the effective conductivity above $10^{-8} \mathrm{~S} / \mathrm{m}$. In Fig. 4 , the effective conductivity component $\bar{k}_{11}$ is plotted as a function of the graphene volume fraction for the values $\Phi_{0}=0.17 \mathrm{eV}, 0.3 \mathrm{eV}, 1.0 \mathrm{eV}$ and without tunneling effect. The aspect ratio is $\eta=50$ and applied electric field $\bar{E}=1.25 \times 10^{-3} \mathrm{~V} / \mathrm{nm}$. It should be noted that for small barrier height $(0.17 \mathrm{eV}$ and $0.3 \mathrm{eV})$, the computation results of electric conductivity taking into account the tunneling effect are much larger than the predictions without tunneling effect, while for $\Phi_{0}=1.0 \mathrm{eV}$, the effective conductivity characteristics exhibit no obvious difference either with or without tunneling effect. The percolation thresholds corresponding to $\Phi_{0}=0.17,0.3,1.0 \mathrm{eV}$ are $0.79 \mathrm{vol} \%, 0.92 \mathrm{vol} \%$ and $1.58 \mathrm{vol} \%$ respectively. We can see from these simulations that the lower the barrier height is, the lower the percolation threshold. Experimental results reporting percolation thresholds for different polymer matrices and graphene types can be found in [7, 45-47].

\subsection{Effect of graphene aspect ratio on the percolation threshold}

Next, we use our numerical model to estimate the percolation threshold $f^{*}$ of the nanocomposite as a function of the graphene volume fraction and aspect ratio for graphene sheets. In Fig. 5, the effective conductivity tensor 


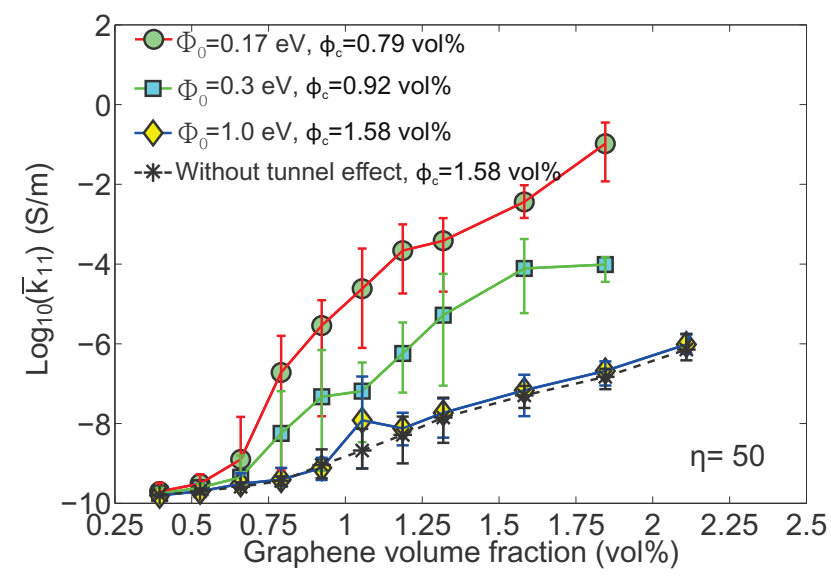

Figure 4: Effective conductivity versus graphene sheets volume fraction for several barrier heights $\Phi_{0}, \bar{E}=1.25 \times 10^{-3} \mathrm{~V} / \mathrm{nm}, \eta=50 ; \phi_{c}$ denotes to the percolation threshold.

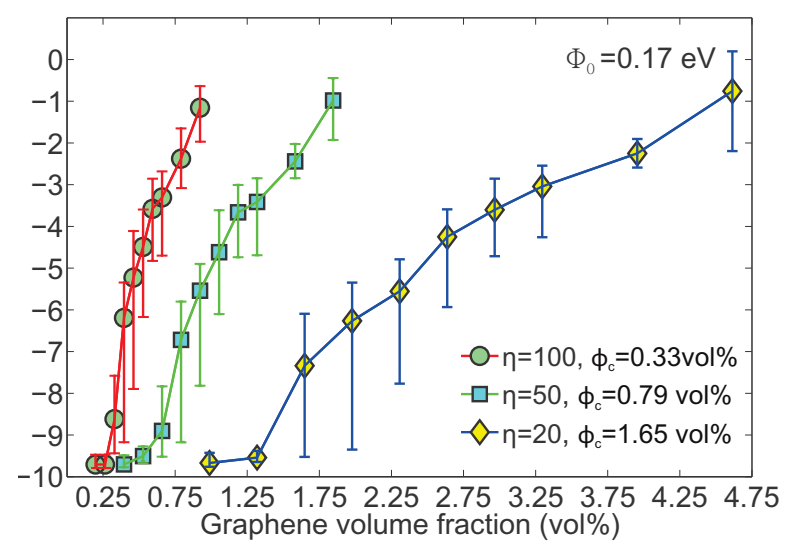

Figure 5: Effective conductivity $\bar{k}_{11}$ as a function of the graphene volume fraction for several graphene aspect ratios $\eta, \bar{E}=1.25 \times 10^{-3} \mathrm{~V} / \mathrm{nm}, \Phi_{0}=0.17 \mathrm{eV} ; \phi_{c}$ denotes to the percolation threshold. 
component $\bar{k}_{11}$ is computed for different aspect ratios $\eta=20,50$ and 100 as a function of volume fraction. The side length of the graphene is fixed, and the various aspect ratios are obtained by changing the thickness of the graphene sheets. For each case, the values are averaged over 30 realizations of random distributions of graphene sheets within the RVE. As the behavior of the composite is nonlinear, the results of conductivity are presented for a fixed electric field $\bar{E}=1.25 \times 10^{-3} \mathrm{~V} / \mathrm{nm}$. The barrier height is $\Phi_{0}=0.17$ $\mathrm{eV}$. According to our numerical simulations, the effective conductivity clearly depends on the aspect ratio, a larger $\eta$ provides lower percolation threshold. The obtained percolation thresholds for $\eta=20,50$, and 100 are $1.65 \mathrm{vol} \%$, $0.79 \mathrm{vol} \%$, and $0.33 \mathrm{vol} \%$ respectively.

\subsection{Effect of alignment of graphene sheets}

Next, we evaluate the effect of alignment of graphene sheets on the effective conductivity of the composite. For this purpose, we consider on one hand a microstructure with graphene sheets whose both positions and orientations are randomly distributed, and on the other hand a microstructure where positions of graphene sheets are randomly distributed but the orientation is fixed. Each point corresponds to the mean value over 30 realizations. Results are presented in Fig. 6. To clearly evidence the anisotropy, we have plotted the three components of the effective conductivity tensor. The parameters are $E=1.25 \times 10^{-3} \mathrm{~V} / \mathrm{nm}, \Phi_{0}=0.17 \mathrm{eV}$ and $\eta=50$.

As expected, the numerical model clearly captures the anisotropic behavior of aligned graphene sheets (see Fig. 6 (b)). Another conclusion is that aligning the graphene sheets does significantly increase either the maximum effective conductivity in the direction normal to the graphene sheets or the percolation threshold, as compared to randomly oriented sheets. However, the increase in conductivity after the percolation threshold is sharper in the case of aligned graphene sheets.

Finally, we depict in Fig. 7 the current density field in the polymer matrix when tunneling effect is taken into account for aligned and randomly oriented graphene sheets, to show the percolation path of electric current in both these configurations. The parameters are $\eta=50$ and $\Phi_{0}=0.17 \mathrm{eV}$, $f=1.05 \mathrm{vol} \%$. The electrical field is applied on the X-direction. 


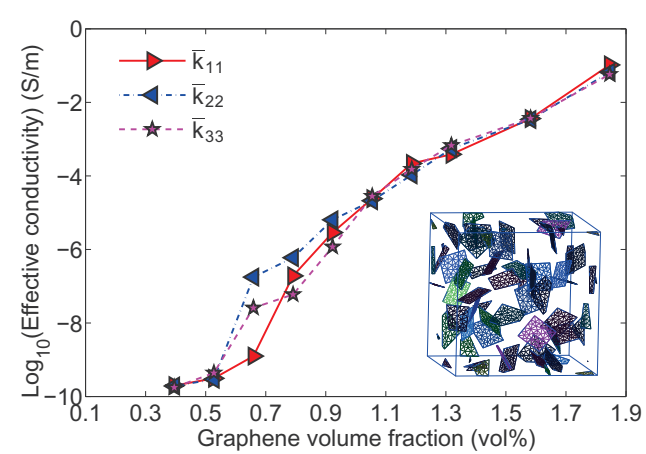

(a)

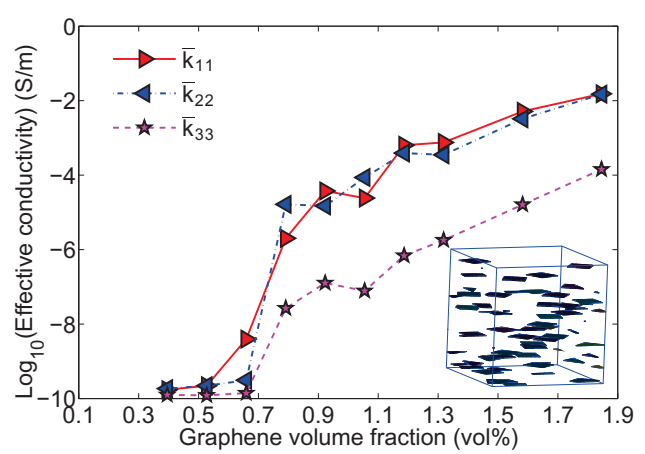

(b)

Figure 6: Effective conductivity tensor components as a function of the graphene volume fraction: (a) random positions and orientations of graphene; (b) random positions and direction of graphene normal to Z-axis.

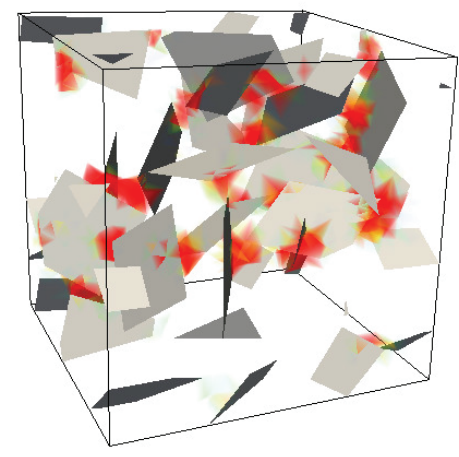

(a)

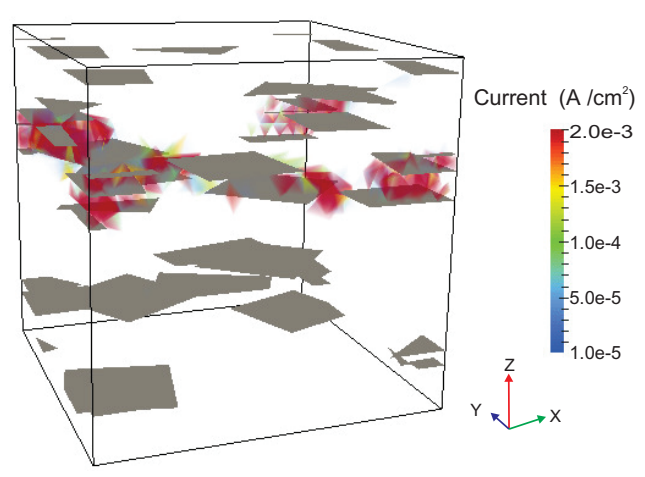

(b)

Figure 7: Current density in the polymer matrix of microstructure with graphene volume fraction $f=1.05$ vol\%: (a) random positions and orientations of graphene; (b) random positions and direction of graphene normal to Z-axis. 


\section{Comparison between numerical and experimental results}

In the following, the obtained numerical results are compared with available experimental data reported by Stankovich [45] and Zhang [48] respectively. The electric conductivity of the polymer matrix is fixed at $10^{-10} \mathrm{~S} / \mathrm{m}$ in the simulation, and the various types of polymer are represented by different choices of barrier height. In these works, the percolation threshold and electric conductivities of graphene reinforced nanocomposites were measured as a function of graphene volume fraction.

Fig. 8 shows the experimental results of electric conductivity of polystyrenegraphene composites as a function of graphene volume fraction measured in [45], where the graphene was prepared by complete exfoliation of graphite and the dispersion of individual graphene sheets at molecular-level. The percolation threshold is exhibited to be $0.1 \mathrm{vol} \%$. The estimated electric conductivities of the composites containing two different aspect ratios of graphene platelets, 200 and 500, obtained from the numerical simulation in our study are also plotted in Fig. 8. Assuming the barrier height to be $0.17 \mathrm{eV}$, we can see that the percolation threshold for composites containing graphene sheets with $\eta=200$ occurred at relatively higher graphene volume fraction, $\phi_{c}=0.2 \mathrm{vol} \%$, than that of the composites containing graphene with $\eta=500$, where $\phi_{c}=0.08$ vol\%. In general, the graphene platelets in the matrix have various aspect ratio due to the preparation method and their possible aggregation during the synthesis. The proposed numerical estimations considering the aspect ratio range of $200-500$ are in reasonable agreement with the experimental data for low graphene volume fraction.

Fig. 9 plots the electric conductivities of polyethylene terephthalategraphene nanocomposites as a function of graphene volume fraction. A high aspect ratio of $\eta=146$ is obtained for graphene sheets from laser granularity analyzer. The experimental measured percolation threshold [48] is about 0.5 vol\%. Similarly, two different aspect ratios of graphene sheets, 50 and 150, were considered in the numerical modeling with the barrier height of $0.3 \mathrm{eV}$, leading to a range of percolation threshold from $0.25 \mathrm{vol} \%$ to $0.8 \mathrm{vol} \%$. The experimentally measured electric conductivities roughly lie between the two numerical curves for the two considered aspect ratios. It should be noted that due to the computational limitations, the contrast of conductivities between graphene and polymer matrix cannot be higher than $10^{16}$. Therefore, we have to take the electric conductivity of polymer matrix as $10^{-10} \mathrm{~S} / \mathrm{m}$, which may be one reason of discrepancy between experimental and numerical results for 


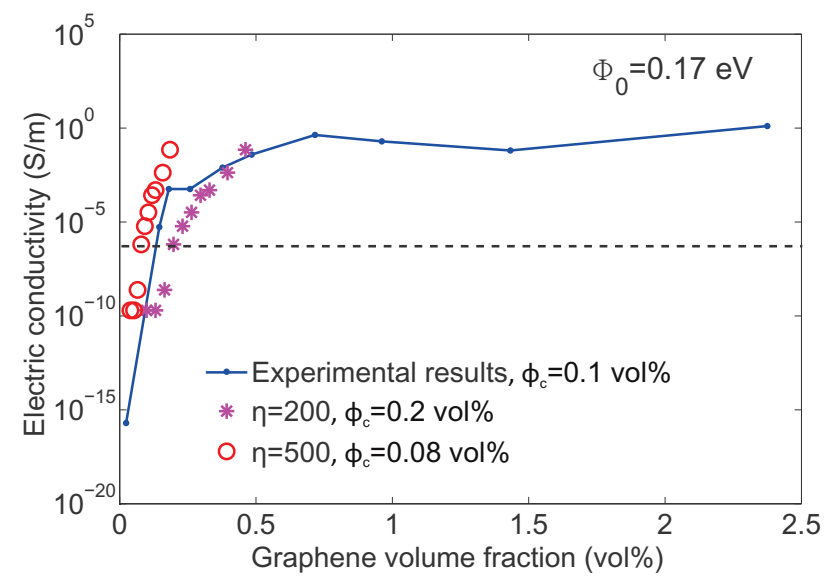

Figure 8: Experimentally measured [45] and predicted electric conductivities of polystyrene-graphene composites with various aspect ratio of graphene sheets $(\eta=$ $200,500) ; \phi_{c}$ denotes to the percolation threshold.

low graphene volume fraction. There are some other reasons which may also bring this discrepancy. Firstly, the dispersion state of graphene is hard to control in the experimental preparation, and the aggregation of graphene in the microstructure may lead to the heterogeneity of the macroscopic property. However, in our present work, the numerical simulation is based on randomly distributed graphene sheets. Moreover, the numerical simulation ignores the defects in the nanocomposite especially the ones on the interface between graphene and polymer. The simulation of electric conductivity at high graphene volume fraction requires large computational complexity due to the refined meshes. For this reason, we did not explored further higher volume fractions, and as the present work focuses on the determination of percolation thresholds. Higher volume fractions could be investigated in future studies. In addition, in the composites the defects like pores are inevitable, but can be optimized by modifying the preparation process. In this work, we did not take the porosity into account because it is not the key influencing factors on the percolation threshold. In addition, we did not consider the nonuniform graphene distribution as the graphene aggregation in this work, but the present framework, described in more details in [38], could be used with larger RVE in future studies to investigate aggregates of graphene platelets. 


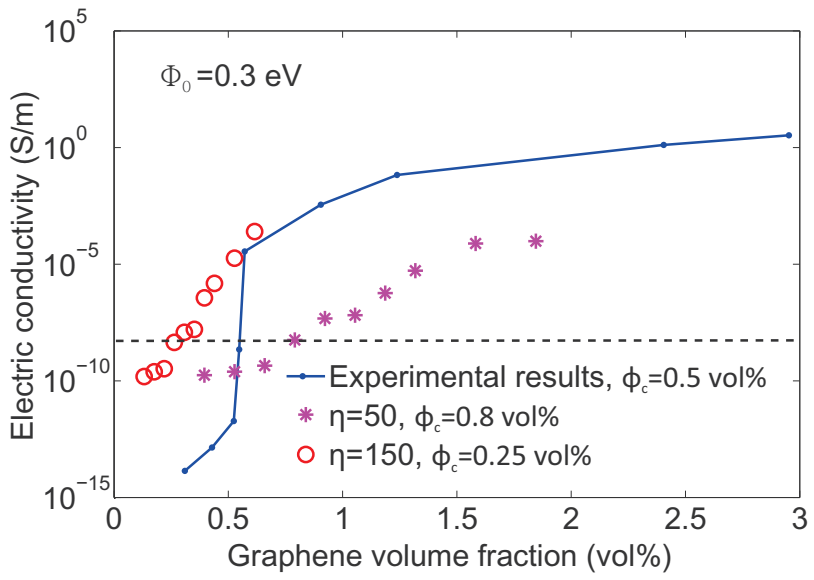

Figure 9: Experimentally measured [48] and predicted electric conductivities of polyethylene terephthalate/graphene nanocomposites with various aspect ratio of graphene sheets $(\eta=50,150) ; \phi_{c}$ denotes to the percolation threshold.

\section{Conclusion}

The numerical simulations show that introducing tunneling effect allows reproducing both low percolation thresholds and nonlinear electrical effects in graphene-reinforced nanocomposites. We report that lower percolation thresholds can be obtained for lower barrier height between the polymer and the graphene sheets and for higher aspect ratios of graphene platelets. The proposed numerical predictions show that aligning the graphene sheets leads to a sharper increase of the effective electric conductivity after the percolation threshold as compared to randomly oriented sheets, but without significant enhancement of percolation thresholds and maximum effective conductivity. Finally, the proposed numerical results were compared to experimental data, showing encouraging agreement and similar trends.

\section{Acknowledgements}

The financial supports from Institut Universitaire de France (IUF) and China Scholarship Council (CSC) are gratefully acknowledged.

\section{References}

[1] L. Britnell, R. M. Ribeiro, A. Eckmann, R. Jalil, B. D. Belle, A. Mishchenko, Y. Kim, R. V. Gorbachev, T. Georgiou, S. V. Moro- 
zov, A. N. Grigorenko, A. K. Geim, C. Casiraghi, A. H. CastroNeto, K. S. Novoselov, Strong light-matter interactions in heterostructures of atomically thin films, Science 340 (6138) (2013) 1311-1314.

[2] M. F. El-Kady, R. B. Kaner, Scalable fabrication of high-power graphene micro-supercapacitors for flexible and on-chip energy storage, Nature Communications 4 (2013) 1475.

[3] K. S. Kim, Y. Zhao, H. Jang, S. Y. Lee, J. M. Kim, K. S. Kim, J. H. Ahn, P. Kim, J. Y. Choi, B. H. Hong, Large-scale pattern growth of graphene films for stretchable transparent electrodes, Nature 457 (7230) (2009) 706.

[4] M. S. Mannoor, H. Tao, J. D. Clayton, A. Sengupta, D. L. Kaplan, R. R. Naik, N. Verma, F. G. Omenetto, M. C. McAlpine, Graphene-based wireless bacteria detection on tooth enamel, Nature Communications 3 (2012) 763 .

[5] W. Guo, C. Cheng, Y. Wu, Y. Jiang, J. Gao, D. Li, L. Jiang, Bio-inspired two-dimensional nanofluidic generators based on a layered graphene hydrogel membrane, Advanced Materials 25 (42) (2013) 6064-6068.

[6] K. S. Novoselov, A. K. Geim, S. V. Morozov, D. Jiang, Y. Zhang, S. V. Dubonos, I. V. Grigorieva, A. A. Firsov, Electric field effect in atomically thin carbon films, science 306 (5696) (2004) 666-669.

[7] J. Liang, Y. Wang, Y. Huang, Y. Ma, Z. Liu, J. Cai, C. Zhang, H. Gao, $\mathrm{Y}$. Chen, Electromagnetic interference shielding of graphene/epoxy composites, Carbon 47 (3) (2009) 922-925.

[8] H. Kim, C. W. Macosko, Processing-property relationships of polycarbonate/graphene composites, Polymer 50 (15) (2009) 3797-3809.

[9] J. Hicks, A. B. A. Ural, A computational study of tunneling-percolation electrical transport in graphene-based nanocomposites, Applied Physics Letters 95 (21) (2009) 213103.

[10] X.-Y. Qi, D. Yan, Z. Jiang, Y.-K. Cao, Z.-Z. Yu, F. Yavari, N. Koratkar, Enhanced electrical conductivity in polystyrene nanocomposites at ultra-low graphene content, ACS applied materials \& interfaces 3 (8) (2011) 3130-3133. 
[11] Y. Wang, J. W. Shan, G. J. Weng, Percolation threshold and electrical conductivity of graphene-based nanocomposites with filler agglomeration and interfacial tunneling, Journal of Applied Physics 118 (6) (2015) 065101.

[12] L. He, S. C. Tjiong, Low percolation threshold of graphene/polymer composites prepared by solvothermal reduction of graphene oxide in the polymer solution, Nanoscale research letters 8 (1) (2013) 132.

[13] P. Fan, L. Wang, J. Yang, F. Chen, M. Zhong, Graphene/poly(vinylidene fluoride) composites with high dielectric constant and low percolation threshold, Nanotechnology 23 (36) (2012) 365702 .

[14] E. Tkalya, M. Ghislandi, R. Otten, M. Lotya, A. Alekseev, P. van der Schoot, J. Coleman, G. de With, C. Koning, Experimental and theoretical study of the influence of the state of dispersion of graphene on the percolation threshold of conductive graphene/polystyrene nanocomposites, ACS Applied Mater. Interfaces 6 (17) (2014) 15113-15121.

[15] C. Gao, S. Zhang, F. Wang, B. Wen, C. Han, Y. Ding, M. Yang, Graphene networks with low percolation threshold in abs nanocomposites: selective localization and electrical and rheological properties, ACS applied materials \& interfaces 6 (15) (2014) 12252-12260.

[16] A. Trionfi, D. Wang, J. Jacobs, L.-S. Tan, R. Vaia, J. Hsu, Direct measurement of the percolation probability in carbon nanofiber-polyimide nanocomposites, Physical review letters 102 (11) (2009) 116601.

[17] F. Du, J. E. Fischer, K. I. Winey, Effect of nanotube alignment on percolation conductivity in carbon nanotube/polymer composites, Physical Review B 72 (12) (2005) 121404.

[18] E. Charlaix, Percolation threshold of a random array of discs: a numerical simulation, Journal of Physics A: Mathematical and General 19 (9) (1986) L533.

[19] M. Mathew, T. Schilling, M. Oettel, Connectivity percolation in suspensions of hard platelets, Physical Review E 85 (6) (2012) 061407. 
[20] M. B. Bryning, M. F. Islam, J. M. Kikkawa, A. G. Yodh, Very low conductivity threshold in bulk isotropic single-walled carbon nanotubeepoxy composites, Advanced materials 17 (9) (2005) 1186-1191.

[21] N. Yousefi, M. M. Gudarzi, Q. Zheng, S. H. Aboutalebi, F. Sharif, Jang-KyoKim, Self-alignment and high electrical conductivity of ultralarge graphene oxide-polyurethane nanocomposites, Journal of Materials Chemistry 22 (25) (2012) 12709-12717.

[22] W. Bauhofer, J. Z. Kovacs, A review and analysis of electrical percolation in carbon nanotube polymer composites, Composites Science and Technology 69 (10) (2009) 1486-1498.

[23] R. Haggenmueller, C. Guthy, J. R. Lukes, J. E. Fischer, K. I. Winey, Single wall carbon nanotube/polyethylene nanocomposites: thermal and electrical conductivity, Macromolecules 40 (7) (2007) 2417-2421.

[24] A. B. Oskouyi, U. Sundararaj, P. Mertiny, Current-voltage characteristics of nanoplatelet-based conductive nanocomposites, Nanoscale Research Letters 9 (1) (2014) 369.

[25] P. Sheng, Fluctuation-induced tunneling conduction in disordered materials, Physical Review B 21 (6) (1980) 2180.

[26] R. H. Otten, P. van der Schoot, Connectivity percolation of polydisperse anisotropic nanofillers, The Journal of chemical physics 134 (9) (2011) 094902.

[27] G. Ambrosetti, C. Grimaldi, I. Balberg, T. Maeder, A. Danani, P. Ryser, Solution of the tunneling-percolation problem in the nanocomposite regime, Physical Review B 81 (15) (2010) 155434.

[28] W. Bauhofer, J. Z. Kovacs, A review and analysis of electrical percolation in carbon nanotube polymer composites, Composites Science and Technology 69 (2009) 1486-1498.

[29] P. Castaneda, J. Willis, The effect of spatial distribution on the effective behavior of composite materials and cracked media, Journal of the Mechanics and Physics of Solids 43 (12) (1995) 1919-1951. 
[30] Z. Fan, F. Gong, S. T. Nguyen, H. M. Duong, Advanced multifunctional graphene aerogel-poly (methyl methacrylate) composites: Experiments and modeling, Carbon 81 (2015) 396-404.

[31] X. Xia, Y. Wang, Z. Zhong, G. J. Weng, A frequency-dependent theory of electrical conductivity and dielectric permittivity for graphenepolymer nanocomposites, Carbon 111 (2017) 221-230.

[32] Y. Wang, G. J. Weng, S. A. Meguid, A. M. Hamouda, A continuum model with a percolation threshold and tunneling-assisted interfacial conductivity for carbon nanotube-based nanocomposites, Journal of Applied Physics 115 (19) (2014) 193706.

[33] C. Grimaldi, I. Balberg, Tunneling and nonuniversality in continuum percolation systems, Physical Review Letters 96 (6) (2006) 066602.

[34] R. H. Otten, P. van der Schoot, Continuum percolation of polydisperse nanofillers, Physical Review Letters 103 (22) (2009) 225704.

[35] R. Hashemi, G. J. Weng, A theoretical treatment of graphene nanocomposites with percolation threshold, tunneling-assisted conductivity and microcapacitor effect in AC and DC electrical settings, Carbon 96 (2016) 474-490.

[36] X. Xia, Z. Zhong, G. J. Weng, Maxwell-Wagner-Sillars mechanism in the frequency dependence of electrical conductivity and dielectric permittivity of graphene-polymer nanocomposites, Mechanics of Materials 109 (2017) 42-50.

[37] A. Manta, M. Gresil, C. Soutis, Predictive model of graphene based polymer nanocomposites: Electrical performance, Applied Composite Materials 24 (2) (2017) 281-300.

[38] X. Lu, J. Yvonnet, F. Detrez, J. Bai, Multiscale modeling of nonlinear electric conductivity in graphene-reinforced nanocomposites taking into account tunnelling effect, Journal of Computational Physics 337 (2017) 116-131.

[39] W. Krauth, Statistical mechanics: algorithms and computations, Vol. 13, OUP Oxford, 2006. 
[40] S. Torquato, Random heterogeneous materials: microstructure and macroscopic properties, Vol. 16, Springer Science \& Business Media, 2013.

[41] J. Yvonnet, Q.-C. He, C. Toulemonde, Numerical modelling of the effective conductivities of composites with arbitrarily shaped inclusions and highly conducting interface, Composites Science and Technology 68 (13) (2008) $2818-2825$.

[42] J. G. Simmons, Electric tunnel effect between dissimilar electrodes separated by a thin insulating film, Journal of Applied Physics 34 (9) (1963) 2581-2590.

[43] R. Rahman, P. Servati, Effects of inter-tube distance and alignment on tunnelling resistance and strain sensitivity of nanotube/polymer composite films, Nanotechnology 23 (5) (2012) 055703.

[44] J. Wang, S. Yu, S. Luo, B. Chu, R. Sun, C.-P. Wong, Investigation of nonlinear i-v behavior of cnts filled polymer composites, Materials Science and Engineering: B 206 (2016) 55-60.

[45] S. Stankovich, D. A. Dikin, G. H. Dommett, K. M. Kohlhaas, E. J. Zimney, E. A. Stach, R. D. Piner, S. T. Nguyen, R. SRuoff, Graphenebased composite materials, nature 442 (7100) (2006) 282-286.

[46] J. Y. Jiang, M. S. Kim, H. M. Jeong, C. M. Shon, Graphite oxide/poly (methyl methacrylate) nanocomposites prepared by a novel method utilizing macroazoinitiator, Composites Science and Technology 69 (2) (2009) 186-191.

[47] S. Ansari, E. P. Giannelis, Functionalized graphene sheet-poly (vinylidene fluoride) conductive nanocomposites, Journal of Polymer Science Part B: Polymer Physics 47 (9) (2009) 888-897.

[48] H.-B. Zhang, W.-G. Zheng, Q. Yan, Y. Yang, J.-W. Wang, Z.-H. Lu, G.-Y. Ji, Z.-Z. Yu, Electrically conductive polyethylene terephthalate/graphene nanocomposites prepared by melt compounding, Polymer 51 (5) (2010) 1191-1196. 